\title{
Aspirin-Triggered Lipoxin and Resolvin E1 Modulate Vascular Smooth Muscle Phenotype and Correlate with Peripheral Atherosclerosis
}

\author{
Karen J. Ho, ${ }^{*}$ Matthew Spite, ${ }^{\dagger}$ \\ Christopher D. Owens, ${ }^{* \neq}$ Hope Lancero, ${ }^{\ddagger}$ \\ Alex H.K. Kroemer, ${ }^{\S}$ Reena Pande, ${ }^{\pi}$ \\ Mark A. Creager, ${ }^{\text {"ा }}$ Charles N. Serhan, ${ }^{\dagger}$ \\ and Michael S. Conte ${ }^{\star \neq}$

\begin{abstract}
From the Division of Vascular and Endovascular Surgery," the Center for Experimental Therapeutics and Reperfusion Injury, ${ }^{\dagger}$ and the Division of Vascular Medicine, "Department of

Medicine, Brigham and Women's Hospital and Harvard Medical School, Boston, Massachusetts; the Laboratory for Accelerated Vascular Research, ${ }^{\ddagger}$ University of California San Francisco, San Francisco, California; and the Transplant Research Center, ${ }^{\S}$ Beth
\end{abstract} \\ Israel Deaconess Medical Center, Boston, Massachusetts
}

Atherosclerosis is a chronic inflammatory disease of the vessel wall. Recent evidence suggests that chronic vascular inflammation ensues as an imbalance between pro- and anti-inflammatory mediators. Recently identified lipid mediators (eg, lipoxins and resolvins) play active roles in promoting the resolution of inflammation. Alterations in vascular smooth muscle cell (VSMC) phenotype, which manifest as a loss of contractile protein expression and increased proliferation and migration, are prominent mechanistic features of both atherosclerosis and restenosis following various interventions (eg, angioplasty and bypass grafting). We sought to determine whether human atherosclerosis is associated with a "resolution deficit" and whether lipoxins and resolvins influence VSMC phenotype. Here we report that plasma levels of aspirin-triggered lipoxin are significantly lower in patients with symptomatic peripheral artery disease than in healthy volunteers. Both aspirintriggered lipoxin and resolvin E1 block platelet-derived growth factor-stimulated migration of human saphenous vein SMCs and decrease phosphorylation of the platelet-derived growth factor receptor- $\beta$. Importantly, receptors for aspirin-triggered lipoxin and resolvin E1 (ALX and ChemR23, respectively) were identified in human VSMCs. Overall, these results demonstrate that stimulatory lipid mediators confer a protective phenotypic switch in VSMCs and elucidate new functions for these mediators in the regulation of SMC biology. These results also suggest that peripheral artery disease is as- sociated with an inflammation-resolution deficit and highlight a potential therapeutic opportunity for the regulation of vascular injury responses. (Am J Pathol 2010, 177:2116-2123; DOI: 10.2353/ajpath.2010.091082)

Chronic inflammation is an underlying component of many disease states including atherosclerosis, Alzheimer's disease, and arthritis. ${ }^{1,2}$ Emerging evidence suggests that the transition from acute to chronic inflammation involves the loss of endogenously operative resolution programs. ${ }^{3}$ The acute inflammatory response develops and actively resolves to re-establish homeostasis and involves the local biosynthesis and actions of lipid autacoids. ${ }^{3}$ While cyclooxygenase (COX) and lipoxygenase (LOX)-derived lipid mediators such as the prostaglandins and leukotrienes have well-established roles in promoting inflammation, ${ }^{4}$ it is now clear that anti-inflammatory and pro-resolving lipid mediators are generated in a temporal and spatial manner to actively turn off inflammation. Among these endogenous pro-resolution mediators are the lipoxins and resolvins. ${ }^{5}$

Lipoxin $A_{4}\left(L X A_{4}\right)$ is generated from arachidonic acid through the sequential action of LOX enzymes. Aspirin acetylation of COX-2 gives rise to epimeric lipoxins, termed aspirin-triggered lipoxin (ATL) in the case of

Supported by National Institutes of Health grants P50-DE016191 (to C.N.S.), HL75771 (to M.S.C., M.A.C., C.D.O.), K23 HL-92163 (to C.D.O.), HL087526 and P20-RR024489 (to M.S.), and the American Vascular Association (to C.D.O.).

K.J.H. and M.S. contributed equally to this work.

Accepted for publication June 16, 2010.

C.N.S. is inventor on patents assigned to Brigham and Women's Hospital and Partners Healthcare on the composition of matter, uses, and clinical development of lipoxins, resolvins, and related compounds. These are licensed for clinical development. C.N.S. also retains founder stock in Resolvyx Pharmaceuticals.

Current address of M.S.: Division of Cardiovascular Medicine, University of Louisville, Louisville, Kentucky.

Address reprint requests to Michael S. Conte, M.D., Professor and Chief, Division of Vascular Surgery, University of California, San Francisco, 400 Parnassus Ave., Suite A581, San Francisco, CA 94143-0222. E-mail: michael.conte@ucsfmedctr.org. 
$\mathrm{LXA}_{4}$, by switching the enzymatic activity from a prostaglandin endoperoxide synthase to a lipoxygenase. ${ }^{5}$ We have previously shown that the endothelium participates in transcellular biosynthesis of ATL through the generation and delivery of $15 R$-hydroxyeicosatetraenoic acid to adhering leukocytes. ${ }^{6}$ Recently, endogenous anti-inflammatory and pro-resolving mediators generated from $\omega-3$ fatty acids, eicosapentaenoic acid and docosahexaenoic acid, were characterized during the resolution phase of acute inflammation, termed resolvins of the E-series and D-series, respectively. ${ }^{5,7,8}$ The complete stereochemistry and receptor-specific bioactions have been characterized for eicosapentaenoic acid -derived resolvin E1 (RvE1), which has potent anti-inflammatory and pro-resolving actions that have been demonstrated in many disease states. ${ }^{5,9}$ Additionally, the protective roles of these mediators were recently demonstrated in murine atherosclerosis, where expression of 12/15 lipoxygenase was associated with atheroprotection and lipoxin and resolvin biosynthesis. These mediators also showed antiinflammatory actions with isolated endothelial cells and macrophages, suggesting that inflammation associated with atherosclerosis ensues partly as a result of the loss of operative pro-resolution pathways. ${ }^{10}$

Recent evidence has highlighted the relationships between inflammation, clinical manifestations of advanced atherosclerosis, and responses to vascular interventions. Inflammatory markers such as high-sensitivity C-reactive protein (hsCRP) are associated with an increased risk of cardiovascular events and with the severity of peripheral arterial disease (PAD). ${ }^{11}$ For example, we have previously found that patients undergoing vein bypass surgery for advanced PAD have elevated preoperative levels of hsCRP and these levels are associated with an increased risk for adverse clinical outcomes and altered graft remodeling. ${ }^{12}$ Exogenous application of human CRP to vascular smooth muscle cells (VSMCs) derived from human saphenous vein increases platelet-derived growth factor (PDGF)stimulated chemotaxis in a PDGFR $\beta$-dependent fashion. ${ }^{13}$ These data and others suggest that VSMCs may integrate local pro- and anti-inflammatory signals in ways that have relevance to lesion development.

Here, we investigated the hypothesis that anti-inflammatory and pro-resolving lipid mediators, lipoxins and resolvins, counteract VSMC responses to the prototypic autocrine/paracrine growth factor PDGF. In addition, we provide further direct evidence to support the conceptual framework that atherosclerosis is the result of a failure of resolution in humans with PAD.

\section{Materials and Methods}

\section{Compounds}

Resolvin E1 (RvE1; 5S,12R,18R-trihydroxy-6Z, 8E, 10E, 14Z, 16E-eicosapentaenoic acid), resolvin D1 (RvD1; 7S, 8R, 17S-trihydroxy-4Z, 9E, 11E, 13Z, 15E, 19Z-docosahexaenoic acid), and aspirin-triggered lipoxin $A_{4}$ (ATL; 5S, 6R, 15R-trihydroxy-7E, 9E, 11Z, 13E-eicosatetraenoic acid) were prepared by total organic synthesis and char- acterized by LC-UV-MS/MS for structural integrity and to establish concentration as described. ${ }^{9,14,15}$

\section{Human Cohort and Measurement of ATL}

Human subjects who participated in this study were recruited from a prospective clinical trial investigating the association of systemic inflammation and insulin resistance on the incidence and prevalence of PAD and vein graft stenosis. Whole blood was collected from a total of 161 patients with PAD (claudication, $n=88$; critical limb ischemia, $n=73$ ) or from controls $(n=38)$ in fasting state after obtaining informed consent and IRB approval from the Brigham and Women's Hospital. Sera were prepared, aliquoted, and stored at $-80^{\circ} \mathrm{C}$. Levels of ATL were assayed in duplicate using a commercially-available enzyme-linked immunosorbent assay kit (Neogen, Lexington, $\mathrm{KY}$ ). This assay has a sensitivity of $0.05 \mathrm{ng} / \mathrm{ml}$. The hsCRP test was determined using an immunoturbidimetric assay on a Hitachi 917 analyzer (Roche Diagnostics, Indianapolis, IN) using reagents and calibrators from Denka Seiken (Niigata, Japan). This assay has a sensitivity of $0.03 \mathrm{mg} / \mathrm{L}$. The day-to-day variabilities of the assay at concentrations of $0.91 \mathrm{mg} / \mathrm{L}, 3.07 \mathrm{mg} / \mathrm{L}$, and $13.38 \mathrm{mg} / \mathrm{L}$ are $2.81,1.61$, and $1.1 \%$, respectively.

\section{Cell Culture}

Primary cultures of human saphenous vein smooth muscle cells (HSVSMCs) were isolated from saphenous vein discarded at the time of bypass operation and maintained in Dulbecco's modified Eagle's medium (Gibco) containing $10 \%$ fetal calf serum, glutamine, and penicillin/streptomycin as described. ${ }^{16}$ Cells were phenotyped using smooth muscle-specific $\alpha$-actin staining, revealing that all cells were smooth muscle cells. Cells isolated from different donors were used between passages 3 and 5 for migration experiments and before passage 7 for all other experiments.

\section{RNA Purification and Gene Expression}

Total RNA was purified from HSVSMCs using Trizol reagent (Invitrogen) according to the manufacturer's instructions. One microgram of RNA was reverse transcribed to $c D N A$ in the presence of RNase inhibitor using the High Capacity cDNA Reverse Transcription Kit (Applied Biosystems) according to the manufacturer's instructions. RT-PCR was performed using Accuprime Taq polymerase (Invitrogen) and gene specific primers for ALX ${ }^{17}$ and chemR23 (forward 5'- GCACAGCATCACTTCTACCACTTTC-3', reverse 5'-CCTCCATTCTCATTCACCGTTATG-3'). GAPDH served as the endogenous control.

\section{Cell Viability and Chemotaxis}

Cells were cultured in low-serum conditions $(0.1 \%-$ $0.5 \%)$ for 36 hours before all experiments. Cell viability was performed using trypan blue exclusion. Chemotaxis 
was assayed using modified Boyden chambers as previously described, ${ }^{13}$ except that PDGF-BB was used at a concentration of $10 \mathrm{ng} / \mathrm{ml}$. Imatinib (Novartis), a tyrosine kinase inhibitor, was used at a concentration of $4 \mu \mathrm{mol} / \mathrm{L}$, which we previously determined to be the $\mathrm{IC}_{50}$ (data not shown). Cells were pretreated with ATL, RvE1, RvD1 (at indicated concentrations), imatinib, or vehicle $(0.1 \%$ EtOH) control for 15 minutes before the addition of PDGF-BB to the bottom wells. All anti-chemotactic compounds were present in both top and bottom wells for the full duration of chemotaxis experiments (6-9 hours). In some experiments, the ALX receptor antagonist, Boc-1 (Sigma; $100 \mu \mathrm{mol} / \mathrm{L}$ ) was added to the cells 15 minutes before the addition of ATL. All treatment conditions were performed in triplicate wells.

\section{Cell Area Measurement}

HSVSMCs were cultured in chamber slides in serum-free media for 36 hours. Cells were then pretreated with 10 $\mathrm{nmol} / \mathrm{L}$ ATL, $10 \mathrm{nmol} / \mathrm{L}$ RvE1, or vehicle $(0.1 \% \mathrm{EtOH})$ for 2 hours followed by the addition of PDGF-BB $(10 \mathrm{ng} / \mathrm{ml})$ for 1 hour. Cells were washed twice in PBS and then fixed in $3.7 \%$ formaldehyde, labeled with Alexa Fluor 488 phalloidin (Invitrogen), and mounted with DAPI containing mounting media (Vectashield). Cell area was determined by outlining the cell dimensions and computing two-dimensional area using National Institutes of Health ImageJ software (http://rsbweb.nih.gov/ij/, last accessed on May 18, 2010).

\section{Immunoprecipitation and Western Blotting}

Whole cell lysates of HSVSMCs were prepared using radioimmunoprecipitation assay buffer and brief sonication. Cleared lysates were separated by SDS-polyacrylamide gel electrophoresis using 4 to $12 \%$ Tris-glycine precast gels (Invitrogen) and transferred to nitrocellulose membranes using a semidry technique. Membranes were blocked in $5 \%$ milk and probed with an anti-human ALX antibody (clone 304405; R \& D), followed by a horseradish peroxidase-conjugated species-specific secondary antibody. For immunoprecipitation experiments, cells were cultured in low serum (0.5\%) for 48 hours, treated with RvE1, RvD1 and ATL (all $10 \mathrm{nmol} / \mathrm{L}$ ) or vehicle for 15 minutes, and stimulated without or with PDGF-BB (10 $\mathrm{ng} / \mathrm{ml}$ ) for 5 minutes. Protein lysates were prepared for immunoprecipitation of total PDGFR- $\beta$ and Western blotting of phosphotyrosine as previously described. ${ }^{13}$ Bound antibody was detected with enhanced chemiluminescent substrate (West Pico, Pierce) according to the manufacturer's instructions.

\section{Flow Cytometry}

For ALX staining, a monoclonal antibody to human ALX (also denoted FPRL-1; R\&D Systems) was used. HSVSMCs were Fc-blocked by treatment with $1 \mu \mathrm{g}$ of human IgG (Sigma) for 15 minutes at room temperature before staining with $0.25 \mu \mathrm{g}$ phycoerythrin-conjugated anti-hu- man FPRL1 antibody (clone 304405) or IgG isotype control (BD Bioscience) for 45 minutes at $4^{\circ} \mathrm{C}$. Cells were washed in PBS and fixed with Cytofix/Cytoperm (BD Bioscience). For ChemR23 staining, HSVSMCs were harvested and cell suspensions were prepared in PBS. Cells were fixed in $2 \%$ paraformaldehyde, permeabilized with $0.5 \%$ saponin, and then labeled with $0.2 \mu \mathrm{g}$ phycoerythrin-conjugated anti-human ChemR23 monoclonal antibody (clone BZ332) in $100 \mu$ l total staining volume according to the manufacturer's instructions (eBioscience). Phycoerythrin-conjugated Rat IgG2a was used as isotype control (eBioscience). After staining, cells were washed twice and resuspended in 1\% paraformaldehyde before FACS analysis. Samples were acquired using a LSR Il flow cytometer (BD Biosciences); data analysis was performed using FlowJo software (Tree Star).

\section{Statistical Analysis}

Experimental data are presented as mean \pm SEM or median and interquartile range. Clinical data are presented as mean \pm SD. Multiple group comparisons were made using one-way analysis of variance followed by Bonferroni's adjustment where appropriate. For non-normally distributed data, nonparametric (Wilcoxon or KruskalWallis) comparisons were made. Spearman correlation coefficient was used for correlation between biomarkers. Multivariable regression was performed using a generalized linear model. In all cases, a $P$ value $<0.05$ was considered to be significant. All analyses were done in SAS 9.2 (SAS Institute, Cary, NC).

\section{Results \\ Circulating ATL Levels Are Decreased in Patients with Peripheral Artery Disease}

Demographic and clinical data on 161 patients with PAD (claudication or critical limb ischemia) and 38 healthy controls are shown in Table 1. Circulating ATL levels were significantly lower in patients with PAD $(1.06 \pm 0.81$ $\mathrm{ng} / \mathrm{ml})$ versus healthy controls $(1.72 \pm 1.08 \mathrm{ng} / \mathrm{ml}, P=$ 0.002 ; Figure 1). No significant difference was observed between the PAD subgroups presenting with claudication $(1.19 \pm 0.9 \mathrm{ng} / \mathrm{ml})$ versus those with critical limb ischemia (0.91 $\pm 0.6, P=0.109$; Figure 1$)$. ATL levels were significantly lower in patients with diabetes mellitus, $(1.34 \pm 0.99 \mathrm{ng} / \mathrm{ml}$ versus $0.87 \pm 0.56 \mathrm{ng} / \mathrm{ml}, P<0.0001)$ and in patients with coronary artery disease $(1.33 \pm 0.93$ $\mathrm{ng} / \mathrm{ml}$ versus $0.99 \pm 0.82 \mathrm{ng} / \mathrm{ml}, P=0.0087)$. Consistent with previous studies (see below), a significant inverse correlation between age and ATL levels was found in this study (correlation coefficient $=-0.1494, P=0.0357$ ). However, in multivariable regression analysis adjusting for age, gender, coronary artery disease, diabetes mellitus, aspirin use, and hsCRP, the difference in ATL levels between PAD and controls remained significant $(P=0.0053)$. 
Table 1. Baseline Clinical Characteristics of Healthy and Peripheral Artery Disease Patients

\begin{tabular}{|c|c|c|c|c|}
\hline & Healthy subjects & Claudication & Critical limb ischemia & $P$ value \\
\hline$N$ & 38 & 88 & 73 & \\
\hline Age $\pm \mathrm{SD}$ (years) & $52.2 \pm 17.5$ & $65.3 \pm 9.5$ & $70.5 \pm 12.2$ & $<0.0001$ \\
\hline Male, $n(\%)$ & $22(57.9 \%)$ & $66(75.0 \%)$ & $45(61.6 \%)$ & 0.0861 \\
\hline Diabetes mellitus, $n(\%)$ & $0(0 \%)$ & $26(29.6 \%)$ & $39(53.4 \%)$ & $<0.0001$ \\
\hline Coronary artery disease, $n(\%)$ & $0(0 \%)$ & 36 (40.9\%) & $47(64.4 \%)$ & $<0.0001$ \\
\hline Ankle-brachial index $\pm S D$ & $1.1 \pm 0.08$ & $0.62 \pm 0.20$ & $0.53 \pm 0.33$ & 0.0001 \\
\hline Body mass index $\pm \mathrm{SD}\left(\mathrm{kg} / \mathrm{m}^{2}\right)$ & $27.2 \pm 4.8$ & $28.5 \pm 6.4$ & $29.2 \pm 6.8$ & 0.2843 \\
\hline Statin, $n(\%)$ & $0(0 \%)$ & $40(48.2 \%)$ & $56(76.7 \%)$ & $<0.0001$ \\
\hline Aspirin use, $n(\%)$ & $8(22.2 \%)$ & $66(79.5 \%)$ & $62(84.9 \%)$ & $<0.0001$ \\
\hline $\mathrm{ATL} \pm \mathrm{SD}(\mathrm{ng} / \mathrm{ml})$ & $1.72 \pm 1.1$ & $1.19 \pm 0.9$ & $0.91 \pm 0.6$ & $<0.0001$ \\
\hline $\mathrm{CRP}(\mathrm{mg} / \mathrm{L})$ & $1.41(0.64-2.4)$ & $2.91(0.96-4.64)$ & $6.56(2.31-19.33)$ & $<0.0001$ \\
\hline
\end{tabular}

\section{Human Saphenous Vein SMCs Express ATL and RvE1 Receptors}

The bioactions of ATL and RVE1 are mediated through specific binding of G-protein coupled receptors termed ALX and chemR23, respectively. ${ }^{9,18}$ To determine whether ATL and RvE1 are candidate signaling molecules for VSMCs, we first determined if their receptors are expressed in isolated HSV SMCs. As shown in Figure 2A, RT-PCR products corresponding to a 390-bp fragment of ALX and a 190-bp fragment of ChemR23 were amplified from HSVSMCs isolated from three different human subjects. Importantly, gene products of both chemR23 and ALX were detected by flow cytometry, where greater than $90 \%$ of the HSVSMCs were positive for ChemR23 (Figure 2B) and ALX (Figure 2C) protein. Of note, expression of ALX was also confirmed by Western analysis (data not shown).

\section{ATL and RVE1 Inhibit PDGF-Stimulated VSMC Chemotaxis}

Addition of ATL or RVE1 alone to HSVSMC cultures did not induce chemotaxis (data not shown), so we next

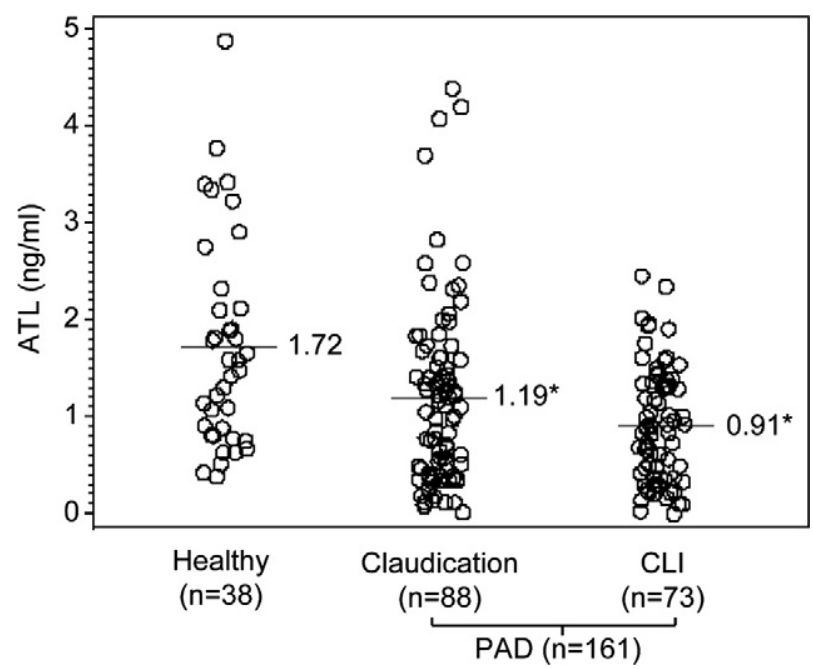

Figure 1. Serum levels of aspirin-triggered lipoxin $A_{4}$ (ATL) inversely correlate with PAD severity in humans. Serum was prepared from healthy volunteers $(n=38)$ and patients with PAD (claudication, $n=88$; critical limb ischemia, $n=73$ ), and ATL levels were analyzed by enzyme-linked immunosorbent assay; *P $P 0.05$. determined if ATL or RvE1 counteract PDGF-stimulated chemotaxis. Pretreatment with ATL resulted in a concentration-dependent $(1 \mathrm{nmol} / \mathrm{L}$ to $500 \mathrm{nmol} / \mathrm{L})$ and statistically significant inhibition of PDGF-BB-stimulated (10 $\mathrm{ng} / \mathrm{ml}$ ) chemotaxis, reaching nearly $40 \%$ inhibition at a dose of $500 \mathrm{nmol} / \mathrm{L}$ (Figure 3A; $P<0.05$ ) with an $\mathrm{IC}_{50}$ value of $2.54 \times 10^{-7} \mathrm{~mol} / \mathrm{L}$. Imatinib $(4 \mu \mathrm{mol} / \mathrm{L})$, a tyrosine-kinase inhibitor, was used as a positive control (Figure 3A). Importantly, the inhibitory effect of ATL was abrogated by the presence of Boc-1 peptide (100 $\mu \mathrm{mol} / \mathrm{L}$ ), a nonselective ALX antagonist, ${ }^{19}$ (Figure 3B) indicating that these ATL responses are likely receptormediated. The methyl ester of RvE1 (which enhances stability of RvE1 in culture medium and was slowly de-esterified to the free acid over the time course of the incubation; data not shown), also decreased PDGFstimulated VSMC chemotaxis in a concentration-dependent manner and was statistically significant at doses as low as $1 \mathrm{nmol} / \mathrm{L}$ (Figure $3 \mathrm{C}$ ). The overall efficacy of RVE1 was greater than that of ATL, with an $I C_{50}$ value of $2.14 \times 10^{-10} \mathrm{~mol} / \mathrm{L}$. Interestingly, docosahexaenoic acid-derived resolvin D1 (RvD1; 10 and $100 \mathrm{nmol} / \mathrm{L}$ ) had no significant effect on VSMC chemotaxis in response to PDGF-BB (data not shown). Importantly, no changes in cell proliferation or viability were observed with treatment of ATL or RvE1 at 10 and 100 $\mathrm{nmol} / \mathrm{L}$ concentrations over 6 to 8 hours as assessed by cell counting and trypan blue exclusion.

To examine if ATL or RVE1 had direct effects on VSMC cytoskeletal arrangement, we quantified cell areas on actin-phalloidin stained cultures. As expected, VSMCs exposed to PDGF-BB exhibit a significant reduction in cell area associated with assumption of a spindle morphology (2374.2 $\pm 400.9 \mu \mathrm{m}^{2}$ control versus $1406.3 \pm$ $289.0 \mu \mathrm{m}^{2}$ PDGF, $\left.P=0.002\right)$. Treatment with ATL significantly increased cell area $\left(2942.2 \pm 458.2 \mu \mathrm{m}^{2}\right.$ ATL versus $1690.8 \pm 255.9 \mu \mathrm{m}^{2}$ vehicle, $P=0.0007$ ); however, VSMCs pretreated with ATL still demonstrated a positive response to PDGF-BB with similar area reduction (ATL $2942 \pm 458.2 \mu \mathrm{m}^{2}$ versus ATL+PDGF $1917.2 \pm$ $484.8 \mu \mathrm{m}^{2}, P=0.0018$ ). Treatment with RvE1 produced similar findings in the cell area assay (not shown). These findings suggest that the effects of ATL and RVE1 on VSMC chemotaxis to PDGF-BB are not completely explained by a disruption of actin microfilament rearrangement to the agonist. 
A

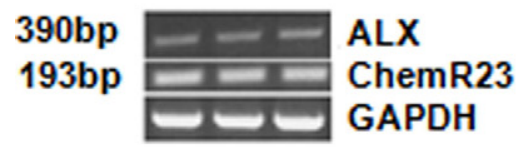

Figure 2. Identification of ALX and ChemR23 in human saphenous vein smooth muscle cells. A: RT-PCR products of ALX and ChemR23 with GAPDH shown as a positive control. Analysis of ChemR23 (B) and ALX (C) expression in HSVSMCs by flow cytometry using phycoerythrin-conjugated anti-ChemR23 and antiALX antibodies, respectively. Results are representative of at least three separate cell donors.
B

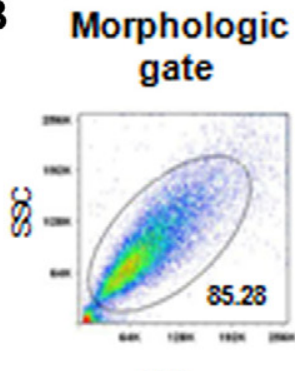

FSC

C Morphologic gate

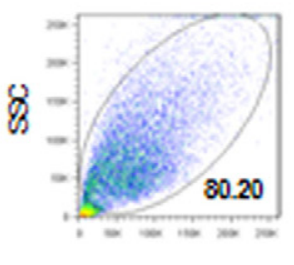

FSC

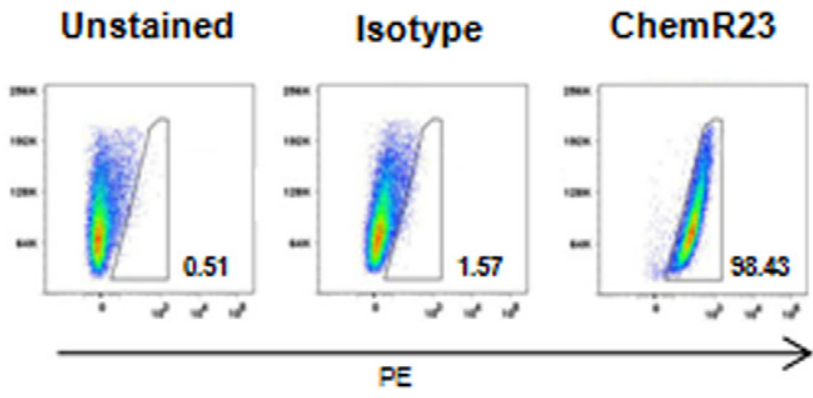

Unstained

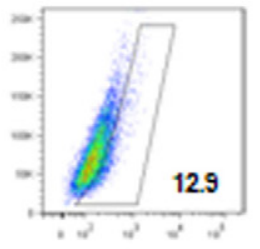

Isotype

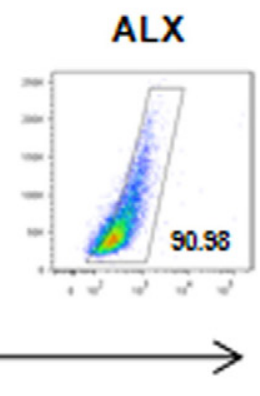

PE

Anti-inflammatory and pro-resolving lipid mediators,

\section{ATL and RVE1 Attenuate PDGF Receptor Activation in HSVSMCS}

To probe the mechanism whereby ATL and RVE1 counter-regulate PDGF-stimulated VSMC chemotaxis, we determined the phosphorylation status of PDGFR- $\beta$ under basal conditions and in the presence of PDGF-BB (10 $\mathrm{ng} / \mathrm{ml})$. Pretreatment of VSMCs with ATL and RvE1 attenuated activation of PDGFR- $\beta$ under basal conditions and following stimulation with PDGF-BB, without decreasing the total amount of detected receptor, at doses of just 10 $\mathrm{nmol} / \mathrm{L}$ (Figure 4). Consistent with the functional responses in modulating PDGF-stimulated chemotaxis, RvD1 did not alter PDGFR- $\beta$ receptor phosphorylation in the presence of PDGF-BB.

\section{Discussion}

The present study documents potent actions of the antiinflammatory and pro-resolving lipid mediators ATL and RvE1 in counter-regulating PDGF-stimulated SMC chemotaxis in human cells. Moreover, we identified the specific receptors for ATL and RVE1 in VSMCs isolated from human saphenous vein. Circulating ATL levels inversely correlated with PAD severity in human subjects even after controlling for other cardiovascular risk factors. In addition, circulating hsCRP values in the PAD cohort were significantly elevated, suggesting that this population has an inflammatory phenotype. Thus, collectively, these studies suggest that resolution failure may have direct relevance for atherosclerosis, and highlight a potential therapeutic opportunity for pro-resolution mediators in controlling vascular inflammation and lesion development.

lipoxins and resolvins, were among the first mediators identified that actively promote the resolution of inflammation, and characterization of their bioactions further demonstrated that resolution is an active process, rather than the result of a passive decrescendo of proinflammatory mediators. ${ }^{5}$ Biosynthesized from arachidonic acid precursor through transcellular biosynthesis in a temporal and spatial manor, lipoxin $A_{4}$ possesses potent antiinflammatory and pro-resolution actions that have been demonstrated in a multitude of acute and chronic inflammatory conditions. ${ }^{5}$ In the presence of aspirin, a $15 R$ epimer of lipoxin $A_{4}$ (ATL) is generated, which, while longer lived than native $L X A_{4}$, shares the potent bioactions of $L X A_{4}$ and binds specifically to the lipoxin receptor ALX with high affinity. ${ }^{18}$ The tissue distribution profile of ALX shows that in both human and mouse, peripheral leukocytes are a major site of ALX expression, with lower amounts in the spleen and lungs. ${ }^{18}$ While $L X A_{4}$ has been shown to modulate the migration of smooth muscle cells in the airway, ${ }^{20}$ this was demonstrated to be mediated through cysteinyl leukotriene receptor antagonism, as ALX was not detected in these cells. Thus, this is the first demonstration that VSMCs express ALX. Similarly to ALX, we also found that the receptor for RvE1, ChemR23, is expressed in human VSMCs. The $\omega$-3-derived RVE1 is a member of a new family of eicosapentaenoic acid -derived resolvins that are enzymatically biosynthesized pro-resolving mediators originally identified in resolving exudates in murine models of self-limited acute inflammation. ${ }^{5,7}$ Like ATL, RvE1 precursor 18R-hydroxyeicosapentaenoic acid (HEPE) is biosynthesized in endothelial cells by acetylated COX-2, which serves as a substrate for adhering leukocytes to generate RvE1. ${ }^{9}$ 


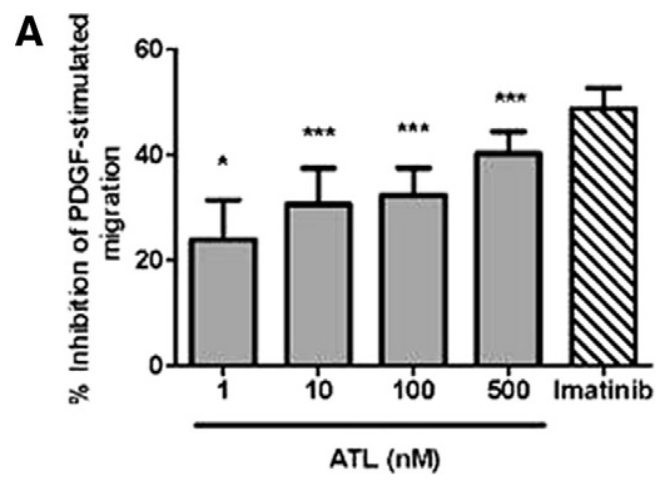

B
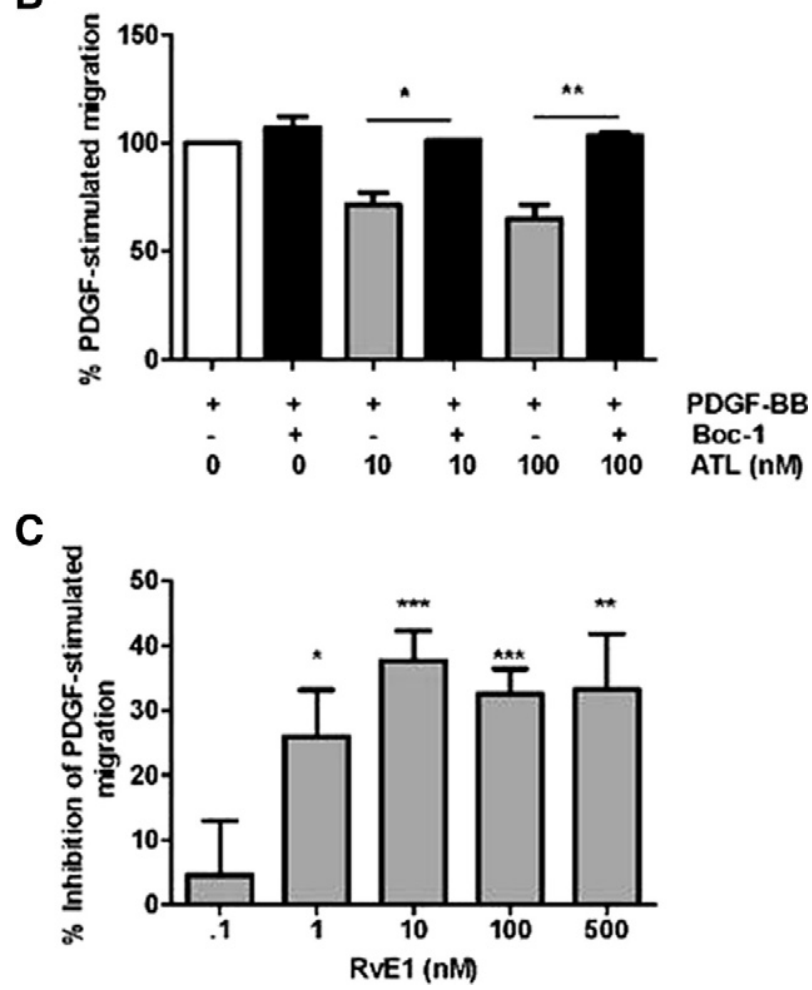

Figure 3. Pretreatment with aspirin-triggered lipoxin $\mathrm{A}_{4}$ (ATL) and resolvin E1 (RvE1) attenuates PDGF-stimulated VSMC migration. A: Inhibition of PDGF-BB (10 ng/ml)-stimulated HSVSMC migration by ATL $(1-500 \mathrm{nmol} / \mathrm{L}$; gray bars); imatinib ( $4 \mu \mathrm{mol} / \mathrm{L}$; hatched bar) is shown as a positive control. B: ALX receptor antagonist Boc- $1(100 \mu \mathrm{mol} / \mathrm{L})$ counteracts the effects of ATL on VSMC migration. C: Inhibition of PDGF-BB-stimulated VSMC migration by $\operatorname{RvE} 1(0.1-500 \mathrm{nmol} / \mathrm{L})$. Results are presented as the mean \pm SEM and are representative of three to six independent experiments. ${ }^{*} P<0.05,{ }^{*} P<0.01$, and ${ }^{* * * * *} P<0.001$ by one-way analysis of variance.

RvE1 binds ChemR23 with a high affinity $\left(K_{d} \approx 11\right.$ $\mathrm{nmol} / \mathrm{L})$ and potently reduces tumor necrosis factor- $\alpha$ stimulated nuclear factor $\kappa \mathrm{B}$ activation in a receptordependent manner. Recently, our laboratory characterized the potent actions of RvE1 in human whole blood and isolated platelets, where RvE1 decreased leukocyte CD18 surface expression and platelet aggregation. ${ }^{21}$ Collectively, the identification of anti-inflammatory/pro-resolving receptors in VSMCs identifies new targets for controlling abnormal SMC responses associated with inflammatory diseases.

The migration and de-differentiation of VSMCs is a characteristic feature of atherosclerotic lesion formation

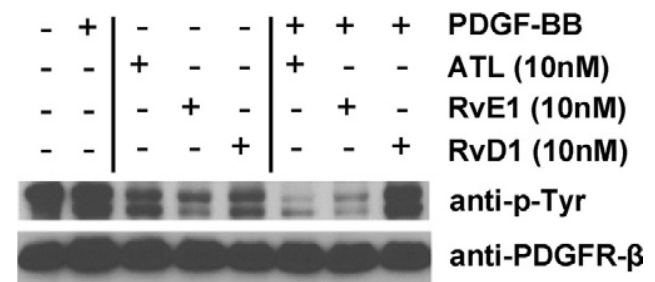

Figure 4. PDGFR- $\beta$ phosphorylation status is modulated by ATL and RvE1. HSVSMCs (in $0.5 \%$ serum) were treated with ATL, RvE1, or RvD1 alone or 15 minutes before the addition of PDGF-BB $(10 \mathrm{ng} / \mathrm{ml})$ and the phosphorylation status of PDGFR- $\beta$ was determined by immunoblot analysis using phosphotyrosine-specific antibodies after immunoprecipitation of total PDGFR- $\beta$ Results are representative of three independent experiments.

and neointima formation following arterial injury and vein bypass grafting. It is becoming increasingly apparent that inflammation is a major component of vascular disease and one of the most prominent biomarkers for inflammatory status is hsCRP. Recently, our laboratory demonstrated that CRP may also play an active role in the vessel wall by stimulating both the expression and activity of the PDGR receptor, as well as the enhancement of VSMC migration, which could have implications for vein graft disease. ${ }^{13}$ Accordingly, elevated hsCRP levels correlate with preoperative PAD severity, as well as adverse postoperative events in a cohort of patients undergoing lower extremity vein bypass surgery. ${ }^{12,22}$ The results of the present study demonstrate that both ATL and RvE1 attenuate PDGF-stimulated VSMC migration and receptor phosphorylation without altering PDGF-R expression levels. This receptor cross talk is consistent with previous reports which showed that lipoxins decrease PDGF-R phosphorylation in mesangial cells. ${ }^{23}$ Of note, ATL and RVE1 decreased PDGF-stimulated VSMC migration at doses as low as $1 \mathrm{nmol} / \mathrm{L}$, while other lipid mediators, such as 11,12-epoxyeicosatrienoic acid, modulate PDGF-stimulated VSMC migration at doses over 1000 times higher. ${ }^{24}$ Of note, the dose range in which ATL counter-regulates VSMC migration is within the range measured in human patients ( $\sim 5 \mathrm{nmol} / \mathrm{L}$ for healthy controls). Thus, ATL and RvE1 are potent counter-regulators of growth-factor related SMC activation. Given the heterogeneity of VSMCs throughout the vascular system, it will be important to determine how VSMCs from different vascular beds respond to ATL and RVE1 and whether differential expression of ATL and RvE1 receptors exists.

Although previous studies have provided valuable information regarding changes in lipid mediator generation with inflammatory pathologies, human data are lacking. Therefore, we sought to establish whether circulating ATL levels correlate with PAD presence and severity in a cross-sectional cohort. We have previously shown that healthy individuals taking aspirin generate ATL in a gender- and age-dependent manner. ${ }^{25,26}$ The results presented here document that PAD is associated with a resolution deficit, where diminished ATL production was observed in PAD patients compared to healthy controls. This is consistent with results from human patients with severe asthma, who also show deficiencies in lipoxin production. ${ }^{27}$ While the independent association be- 
tween ATL and PAD was maintained in a multivariable model, there was significant interaction between plasma ATL values and patient age. Given that we did not have an age-matched control population, the conclusions drawn must be tempered. However, in the multivariable model, ATL was the strongest independent predictor of the presence of PAD. Of note, statins have also been shown to increase myocardial production of ATL in rats, suggesting that some of the anti-inflammatory actions of statins may be the result of increased ATL production. ${ }^{28}$ This and other pathways (ie, cytochrome P450) may account for ATL biosynthesis in patients not taking aspirin. ${ }^{29}$ With respect to atherosclerosis, recent reports from our laboratory indicated that lipoxin and resolvin production are associated with atheroprotection in murine models of atherosclerosis. Genetic manipulation of one enzyme necessary for lipoxin and resolvin biosynthesis (12/ 15-LOX) demonstrated that this pathway was protective against atherosclerotic lesion formation. Mass spectrometry-based lipid mediator lipidomic analysis revealed that both lipoxins and resolvins were generated by macrophages isolated from atherosclerotic mice and that their levels coincided with 12/15-LOX gene dosage. In vitro, both lipoxins and resolvins decreased proinflammatory cytokine generation by macrophages and modulated adhesion receptor expression on endothelial cells. ${ }^{10}$ Of interest, docosahexaenoic acid-derived resolvin D1 (RvD1) showed potent anti-inflammatory actions with isolated macrophages and endothelial cells, while RvD1 was not a regulator of VSMC migration in the present studies. Thus, these and related studies highlight the distinct cell type- specific actions of resolvins in complex inflammatory diseases.

In contrast to the beneficial effects of lipoxins and resolvins in vascular pathologies, proinflammatory lipid mediators such as the leukotrienes have been implicated in the promotion of atherosclerosis. In line with our current studies identifying the bioactions of lipoxins and resolvins on VSMCs, enzymes required for the biosynthesis for leukotrienes were recently identified in VSMCs. ${ }^{30}$ In addition, the receptor for leukotriene $\mathrm{B}_{4}(\mathrm{BLT}-1)$ was identified on human VSMCs and co-localized with VSMCs from human atherosclerotic plaques. Interestingly, leukotriene $\mathrm{B}_{4}$ stimulated VSMC migration in vitro, and inhibition of the BLT-1 receptor protected rabbits from intimal hyperplasia. ${ }^{31}$ These studies lend further support to the concept that an imbalance of pro- versus anti-inflammatory lipid mediators sets the stage for vascular inflammation and expand our current understanding of the role of lipid mediators in controlling a multitude of biological responses beyond those of only myeloid cells.

In summation, the present studies highlight the novel role of lipoxins and resolvins in counter-regulating PDGF-receptor activation and subsequent VSMC migration. The relative deficiency in circulating ATL levels in human patients with PAD suggests that altered resolution is associated with atherosclerosis, and that ATL may serve as a valuable biomarker for progressive vascular inflammation. In addition, these studies suggest a novel therapeutic strategy for controlling VSMC migration which is central to both atherosclerosis and treatment failures following angioplasty and bypass surgery.

\section{Acknowledgment}

We acknowledge the assistance of Nathanael Hevelone, M.P.H., for statistical review.

\section{References}

1. Hansson GK, Libby P: The immune response in atherosclerosis: a double-edged sword. Nat Rev Immunol 2006, 6:508-519

2. Perry VH, Cunningham C, Holmes C: Systemic infections and inflammation affect chronic neurodegeneration. Nat Rev Immunol 2007, $7: 161-167$

3. Serhan CN, Brain SD, Buckley CD, Gilroy DW, Haslett C, O'Neill LA, Perretti M, Rossi AG, Wallace JL: Resolution of inflammation: state of the art, definitions and terms. FASEB J 2007, 21:325-332

4. Yedgar S, Krimsky M, Cohen Y, Flower RJ: Treatment of inflammatory diseases by selective eicosanoid inhibition: a double-edged sword? Trends Pharmacol Sci 2007, 28:459-464

5. Serhan CN, Chiang N, Van Dyke TE: Resolving inflammation: dual anti-inflammatory and pro-resolution lipid mediators. Nat Rev Immunol 2008, 8:349-361

6. Claria J, Serhan CN: Aspirin triggers previously undescribed bioactive eicosanoids by human endothelial cell-leukocyte interactions. Proc Natl Acad Sci USA 1995, 92:9475-9479

7. Serhan CN, Clish CB, Brannon J, Colgan SP, Chiang N, Gronert K Novel functional sets of lipid-derived mediators with antiinflammatory actions generated from omega-3 fatty acids via cyclooxygenase 2-nonsteroidal antiinflammatory drugs and transcellular processing. J Exp Med 2000, 192:1197-1204

8. Serhan CN, Hong S, Gronert K, Colgan SP, Devchand PR, Mirick G, Moussignac RL: Resolvins: a family of bioactive products of omega-3 fatty acid transformation circuits initiated by aspirin treatment that counter proinflammation signals. J Exp Med 2002, 196:1025-1037

9. Arita M, Bianchini F, Aliberti J, Sher A, Chiang N, Hong S, Yang R, Petasis NA, Serhan CN: Stereochemical assignment, antiinflammatory properties, and receptor for the omega-3 lipid mediator resolvin E1. J Exp Med 2005, 201:713-722

10. Merched AJ, Ko K, Gotlinger KH, Serhan CN, Chan L: Atherosclerosis: evidence for impairment of resolution of vascular inflammation governed by specific lipid mediators. FASEB J 2008, 22:3595-3606

11. Ridker PM: Clinical application of C-reactive protein for cardiovascular disease detection and prevention. Circulation 2003, 107:363-369

12. Owens CD, Ridker PM, Belkin M, Hamdan AD, Pomposelli F, Logerfo F, Creager MA, Conte MS: Elevated C-reactive protein levels are associated with postoperative events in patients undergoing lower extremity vein bypass surgery. J Vasc Surg 2007, 45:2-9

13. Ho KJ, Owens CD, Longo T, Sui XX, Ifantides C, Conte MS: C-reactive protein and vein graft disease: evidence for a direct effect on smooth muscle cell phenotype via modulation of PDGF receptor-beta. Am J Physiol Heart Circ Physiol 2008, 295:H1132-H1140

14. Sun YP, Oh SF, Uddin J, Yang R, Gotlinger K, Campbell E, Colgan SP, Petasis NA, Serhan CN: Resolvin D1 and its aspirin-triggered 17R epimer: stereochemical assignments, anti-inflammatory properties, and enzymatic inactivation, J Biol Chem 2007, 282:9323-9334

15. Serhan CN, Lu Y, Hong S, Yang R: Mediator lipidomics: search algorithms for eicosanoids, resolvins, and protectins. Methods Enzymol 2007, 432:275-317

16. Wang GJ, Sui XX, Simosa HF, Jain MK, Altieri DC, Conte MS: Regulation of vein graft hyperplasia by survivin, an inhibitor of apoptosis protein. Arterioscler Thromb Vasc Biol 2005, 25:2081-2087

17. Bonnans C, Fukunaga K, Levy MA, Levy BD: Lipoxin A(4) regulates bronchial epithelial cell responses to acid injury. Am J Pathol 2006, 168:1064-1072

18. Takano T, Fiore S, Maddox JF, Brady HR, Petasis NA, Serhan CN: Aspirin-triggered 15-epi-lipoxin A4 (LXA4) and LXA4 stable ana- 
logues are potent inhibitors of acute inflammation: evidence for antiinflammatory receptors. J Exp Med 1997, 185:1693-1704

19. John CD, Sahni V, Mehet D, Morris JF, Christian HC, Perretti M, Flower RJ, Solito E, Buckingham JC: Formyl peptide receptors and the regulation of $\mathrm{ACTH}$ secretion: targets for annexin $\mathrm{A} 1$, lipoxins, and bacterial peptides. FASEB J 2007, 21:1037-1046

20. Parameswaran K, Radford K, Fanat A, Stephen J, Bonnans C, Levy BD, Janssen LJ, Cox PG: Modulation of human airway smooth muscle migration by lipid mediators and Th-2 cytokines. Am J Respir Cell Mol Biol 2007, 37:240-247

21. Dona M, Fredman G, Schwab JM, Chiang N, Arita M, Goodarzi A, Cheng G, von Andrian UH, Serhan CN: Resolvin E1, an EPA-derived mediator in whole blood, selectively counterregulates leukocytes and platelets. Blood 2008, 112:848-855

22. Owens CD, Rybicki FJ, Wake N, Schanzer A, Mitsouras D, GerhardHerman MD, Conte MS: Early remodeling of lower extremity vein grafts: inflammation influences biomechanical adaptation. J Vasc Surg 2008, 47:1235-1242

23. McMahon B, Mitchell D, Shattock R, Martin F, Brady HR, Godson C: Lipoxin, leukotriene, and PDGF receptors cross-talk to regulate mesangial cell proliferation. FASEB J 2002, 16:1817-1819

24. Sun J, Sui X, Bradbury JA, Zeldin DC, Conte MS, Liao JK: Inhibition of vascular smooth muscle cell migration by cytochrome p450 epoxygenase-derived eicosanoids. Circ Res 2002, 90:1020-1027

25. Chiang N, Bermudez EA, Ridker PM, Hurwitz S, Serhan CN: Aspirin triggers antiinflammatory 15 -epi-lipoxin A4 and inhibits thromboxane in a randomized human trial. Proc Natl Acad Sci USA 2004, 101: $15178-15183$
26. Chiang N, Hurwitz S, Ridker PM, Serhan CN: Aspirin has a genderdependent impact on antiinflammatory 15-epi-lipoxin A4 formation: a randomized human trial. Arterioscler Thromb Vasc Biol 2006, 26: e14-e17

27. Planaguma A, Kazani S, Marigowda G, Haworth O, Mariani TJ, Israel E, Bleecker ER, Curran-Everett D, Erzurum SC, Calhoun WJ, Castro M, Chung KF, Gaston B, Jarjour NN, Busse WW, Wenzel SE, Levy BD: Airway lipoxin A4 generation and lipoxin A4 receptor expression are decreased in severe asthma. Am J Respir Crit Care Med 2008, 178:574-582

28. Birnbaum Y, Ye Y, Lin Y, Freeberg SY, Nishi SP, Martinez JD, Huang $\mathrm{MH}$, Uretsky BF, Perez-Polo JR: Augmentation of myocardial production of 15-epi-lipoxin-a4 by pioglitazone and atorvastatin in the rat. Circulation 2006, 114:929-935

29. Claria J, Lee MH, Serhan CN: Aspirin-triggered lipoxins (15-epi-LX) are generated by the human lung adenocarcinoma cell line (A549)neutrophil interactions and are potent inhibitors of cell proliferation. Mol Med 1996, 2:583-596

30. Qu H, Straat K, Rahbar A, Wan M, Soderberg-Naucler C, Haeggstrom JZ: Human CMV infection induces 5-lipoxygenase expression and leukotriene B4 production in vascular smooth muscle cells. J Exp Med 2008, 205:19-24

31. Back M, Bu DX, Branstrom R, Sheikine $Y$, Yan ZQ, Hansson GK: Leukotriene B4 signaling through NF-kappaB-dependent BLT1 receptors on vascular smooth muscle cells in atherosclerosis and intimal hyperplasia. Proc Natl Acad Sci USA 2005, 102: 17501-17506 